\title{
ARTICLE Nicotine effects on associative learning in human non-smokers
}

\author{
Britta Hahn ${ }^{1}$, Ashleigh K. Wells ${ }^{1}$, Agatha Lenartowicz ${ }^{2}$ and Marie B. Yuille ${ }^{1}$
}

Tobacco smoking is the most common preventable cause of death in the US. Nicotine is considered the primary constituent responsible for tobacco addiction. Its paradoxically high abuse potential may reflect behavioral control by drug-associated stimuli, which appears to play a larger role for tobacco dependence than for other abused drugs. We tested a potential explanation, hypothesizing that nicotine enhances associative learning, the mechanism underlying the conditioning of drug-associated stimuli. Thirty-two non-smokers were exposed to transdermal nicotine $(7 \mathrm{mg} / 24 \mathrm{~h})$ and placebo in a double-blind cross-over study and tested with behavioral paradigms designed to isolate incidental stimulus-stimulus or stimulus-response learning. The stop signal task required speeded gender judgments of face stimuli. A tone signaled when to withhold the response. Unbeknownst to participants, some faces were always paired with stop trials. Nicotine enhanced the facilitation of stop-responses to these stimuli, and the slowing of go-responses when previously stop-associated stimuli were paired with go trials, indicating stronger associations between paired stimuli and the stop signal/stop response. Another task required feedback-based learning of associations between pairs of shape stimuli. Five pairs were made from either ten different stimuli, or from different combinations of two identical sets of five stimuli with correct associations depending on contextual information. Nicotine increased incorrect choices of stimuli that were associated in a different context, indicating stronger stimulus-stimulus associations at the expense of flexible context-adaptive behavior. The results indicate that nicotine can enhance incidental associative learning, a mechanism that may promote the formation of smoking-associated stimuli and cue-controlled drug-taking.

Neuropsychopharmacology (2018) 43:2190-2196; https://doi.org/10.1038/s41386-018-0183-9

\section{INTRODUCTION}

Tobacco smoking is the leading cause of preventable disease and death in the US [1], and among the top two world-wide [2]. An estimated $20 \%$ of the US adult population are current smokers [3]; over $90 \%$ of quit attempts fail before six months [4]. The primary constituent responsible for tobacco addiction is nicotine [5].

There is an unresolved paradox with regards to nicotine dependence: The prevalence, abuse liability, and relapse rate of tobacco smoking is comparable to or exceeds that of other drugs of abuse $[6,7]$, but the primary reinforcing effects of nicotine are weak. Rats strongly prefer cocaine over nicotine [8], mirroring their euphorogenic effects in humans. Nicotine is not as readily selfadministered by animals as other abused drugs, and displays a flatter inverted U-shaped dose-response curve $[9,10]$. In humans, nicotine pretreatment even at large doses causes only small smoking reductions [11, 12], while denicotinized cigarettes maintain a level of smoking, elicit pleasant subjective effects, and decrease anxiety and craving in smokers [13-15]. Analogously, nicotine self-administration by animals can be remarkably resistant to extinction upon vehicle substitution [16]. The relative ineffectiveness of nicotine replacement in quit attempts [17] further supports the view that acute pharmacological effects cannot fully explain nicotine's dependent use.

A potential explanation of this paradox is that environmental and physiological stimuli associated with nicotine intake exert powerful control over tobacco consumption $[18,19]$. Exposure of smokers to smoking-associated stimuli has been shown to increase craving [20-22], evoke autonomic responses [23-25], capture attention [26-28], activate specific brain regions [29-31], support smoking-behavior, and facilitate relapse [20, 32, 33]. Animal self-administration studies pairing discrete or contextual stimuli with nicotine infusions confirmed that such stimuli promote the acquisition of nicotine seeking, slow its extinction, reinstate it $[18,34,35]$, and act synergistically with nicotine in maintaining self-administration [36]. Remarkably, once nicotine self-administration is acquired with accompanying cues, the cues appear to exert more profound control over nicotine-seeking than nicotine itself [18].

Neutral stimuli become smoking-associated stimuli via associative learning. Through repeated temporal pairing, stimulus-stimulus and stimulus-response associations are formed. Primary reinforcing effects of nicotine become associated with environmental cues, smoking paraphernalia or internal sensations (stimulus-stimulus), and with the drug taking routine (stimulus-response). These associations can be formed with or without awareness, but usually in an incidental, non-deliberate manner and not based on higher cognitive functions involving abstraction or symbolization. Examples of stimulus-stimulus associations are the pairing of smoking-induced throat irritation [19] or a typical smoking environment (e.g., the car) with nicotine's mild euphorogenic effects. A stimulus-response association would be the pairing of nicotine effects, or an associated environment, with the lighting-up routine.

Drug-associated stimuli may be of particular importance to tobacco dependence because of the sheer number of cue

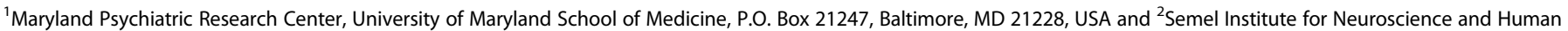
Behavior, University of California Los Angeles, Los Angeles, CA, USA

Correspondence: Britta Hahn (bhahn@som.umaryland.edu)

Received: 8 May 2018 Revised: 27 July 2018 Accepted: 8 August 2018

Published online: 17 August 2018 
pairings, which is larger than for other abused drugs [37]. However, nicotine also differs from other drugs in its ability to directly modulate synaptic plasticity and hippocampus-dependent learning. Nicotine can promote the induction of long-term potentiation and enhances contextual fear conditioning in rats via nicotinic acetylcholine receptors located in the hippocampus, a structure involved in the formation of drug-cue and drug-context associations [38-41]. Thus, there is evidence to suggest that nicotine may facilitate associative learning processes. By promoting the conditioning of smoking-associated stimuli, this may augment conditioned stimulus control over tobacco consumption above and beyond that seen with other drugs of abuse, and explain nicotine's paradoxically high abuse potential. Thus, the aim of the present proof of principle study was to test the hypothesis that nicotine can potentiate associative learning in humans. Such demonstration may lead to a broadening of our understanding of the mechanisms driving nicotine dependence.

\section{MATERIALS AND METHODS}

Subjects

Thirty-two healthy non-smokers completed the study (15 female; 13 African American, 17 Caucasian, 2 Asian). Participants were 22-54 years of age (mean \pm stdev: $33.6 \pm 10.5$ ) with $12-22$ years of education $(15.6 \pm 2.5)$. They were recruited from the local community through internet advertising, flyers and referrals, and gave written informed consent for a protocol approved by the University of Maryland Baltimore Institutional Review Board. Because a proof of principle that nicotine can facilitate human associative learning depends on studying organisms devoid of neuroadaptive changes by chronic nicotine exposure, all participants were current non-smokers, had never been dependent smokers, and had no exposure to tobacco products or vaping within the last year. Only three participants had ever smoked any tobacco in their lifetime (see Supplementary Materials for details).

Procedures

During an initial visit, participants were screened for eligibility and trained on the associative learning tasks to be performed in the later test sessions. Training of the Stop Signal task consisted of two 100-trial blocks in which no stimuli were systematically paired with stop or go trials. Training of the Conditional association learning (CAL) task consisted of 20 trials of the Unique and
Crossed task each. The experimenter was careful to ensure that all participants understood the rules governing stimulus matching in the Crossed task (see below).

Two test sessions followed, scheduled with two to six intermediate days. In one session, participants received a transdermal nicotine patch $\left(7 \mathrm{mg} / 24 \mathrm{~h}\right.$; Nicoderm $\mathrm{CQ}^{\circ}$, GlaxoSmithKline), in the other a placebo patch. The sequence of patch conditions was counterbalanced between participants. Participants and investigators were blind with regard to patch condition, although some participants may have guessed the test sequence based on subjective nicotine effects (see Supplementary Materials for details on the blinding procedures and subjective side effects of nicotine).

Each test session took approximately $7 \mathrm{~h}$ (more details in the Supplement). Study patch application was followed by a drugabsorption period, during which participants were permitted to read or use the internet. $5 \mathrm{~h}$ after patch administration, at which time nicotine blood concentrations were expected to have plateaued, testing began. The Stop Signal task was always performed first and the CAL task second.

\section{Equipment}

Tasks were performed on a desktop PC and presented on a 19-inch 5:4 IPS LCD monitor with a screen resolution of $1280 \times$ 1024 and a $60 \mathrm{~Hz}$ refresh rate. Responses were recorded using a standard keyboard and mouse. The Stop Signal task was implemented in Matlab using the Psychophysics Toolbox extensions. Stop signals were presented via Sony MDRZX100 headphones. The CAL task was implemented in E-Prime 2.0.

\section{Task paradigms}

Stop signal task. The paradigm was based on Lenartowicz et al. [42] and Verbruggen and Logan [43] (Fig. 1). A series of face stimuli (50\% male, $50 \%$ female), selected from a collection compiled by Neal Cohen at the University of Illinois [44], was presented against a black background. Each face was presented for $1000 \mathrm{~ms}$, preceded by a $500 \mathrm{~ms}$ white fixation cross, and followed by a variable intertrial interval of $500-4000 \mathrm{~ms}(1000 \mathrm{~ms}$ average; following a continuous exponential distribution) during which the screen remained blank. Participants made gender judgments about the faces by pressing either the $<$ (male) or $>$ (female) key of the keyboard with their index and middle finger as quickly as possible.

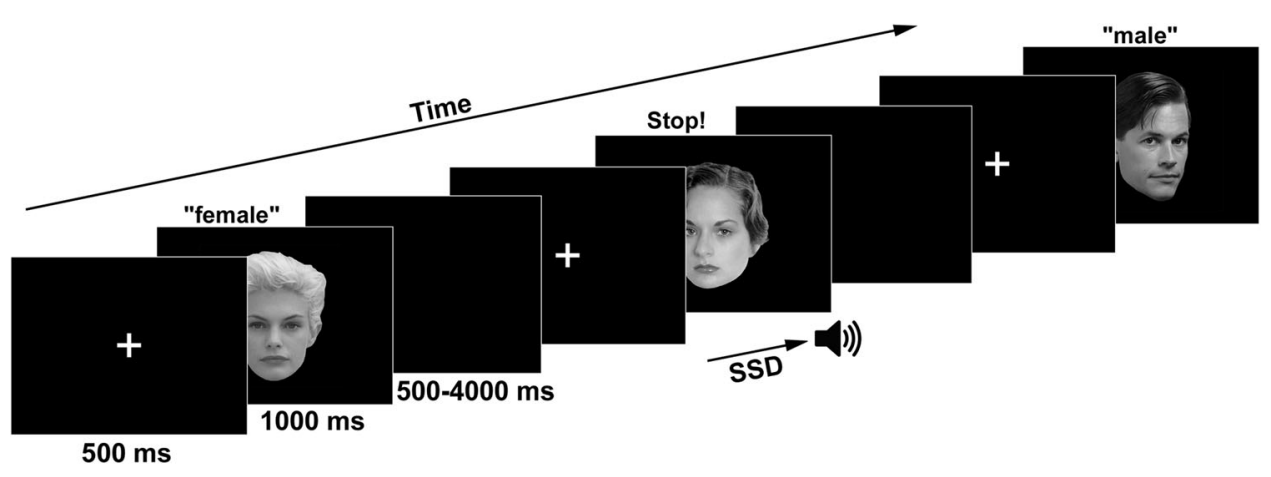

\begin{tabular}{|c|c|c|c|}
\hline Stimulus type & Training phase & Transfer phase & Trials per block \\
\hline go/stop - go/stop & go or stop & go or stop & 32 \\
\hline go/stop - go & go or stop & go & 16 \\
\hline stop - go & stop & go & 12 \\
\hline go - go & go & go & 24 \\
\hline go - stop & go & stop & 16 \\
\hline
\end{tabular}

Fig. 1 Screen displays in the Stop signal task. The stimuli are shown enlarged relative to the size of the screen for illustrative purposes. Three trials are shown: two go trials and one stop trial. The table lists all possible stimulus types as described in the Methods 
On $24 \%$ of trials, a $900 \mathrm{~Hz}$ tone indicated that the response should be withheld (stop trials). The tone was presented for 500 ms with a variable stop signal delay (SSD) relative to the onset of the face stimulus. A staircase procedure was aimed at achieving $50 \%$ probability of stopping successfully [45]: SSD increased by 50 ms after a successful stop and decreased by $50 \mathrm{~ms}$ after a stop failure, thus making stopping more difficult or easier on the next stop trial (note that the stop signal was always presented after face stimulus onset). To minimize SSD predictability, two staircases with different starting-SSDs (250 and $300 \mathrm{~ms}$ ) were initialized and presented interleaved.

The task consisted of ten 100-trial blocks. For each staircase, the average SSD over the last 10 trials of a block was used as the starting SSD for the next block. The first six blocks made up the training phase, followed by a brief break while a new script was initialized. The next two blocks still followed the contingencies of the training phase, but in the last two blocks (transfer phase), contingencies changed (see below). The stimulus set consisted of 50 faces, each presented twice per block.

Unbeknownst to participants, 6 of the 50 faces (12 trials per block) were always paired with stop trials in the training phase, but only with go trials in the transfer phase ("stop-go" stimuli). The main prediction was that, over time, the association with stop trials would facilitate the stop response to these stimuli, but cause interference, reflected by slower go reaction times (goRTs), when they were subsequently paired with go trials. Another 12 faces were paired with only go trials in both phases ("go-go" stimuli); this association was expected to facilitate the go response, as reflected by faster goRTs. Another 8 faces were randomly paired with stop trials or go trials $(25: 75 \%$ chance) during training, but only with go trials in the transfer phase ("go/stop-go" stimuli). These stimuli enabled the comparison of goRTs in the transfer phase between stimuli that had previously been paired with only stop trials or only go trials on the one hand, and stimuli that had not been systematically paired with either on the other hand.

Sixteen faces were randomly paired with stop or go trials ( $25: 75 \%$ chance) in both phases ("go/stop-go/stop" stimuli), and eight faces were paired with go trials in the training phase but with stop trials in the transfer phase ("go-stop" stimuli), to create a $24 \%$ stop ratio overall. The table incorporated in Fig. 1 provides an overview of all stimulus types, which were presented pseudorandomly with the constraint that no stimulus repeated on consecutive trials. Importantly, each stimulus type that involved stop-trials, i.e., (1) stimuli associated with stop trials only, and (2) stimuli associated with either stop or go trials, had their own two staircases with the same two starting values in each phase, thus allowing us to test the effects of the systematic pairing with stop trials on SSD.

We predicted that over the course of the training phase the average SSD for "stop-go" stimuli (paired with stop trials in the training phase) would increase relative to "go/stop-go" stimuli (not systematically paired with stop or go trials in the training phase), reflecting facilitation of the stop response to stopassociated stimuli. We hypothesized that this increase would be greater in the presence of nicotine, reflecting enhanced formation of associations between the paired stimuli and the stop signal or the stop response (stimulus-stimulus and stimulus-response associations cannot be distinguished in this paradigm).

For the transfer phase, we predicted that goRTs would be slowed for "stop-go" relative to "go/stop-go" stimuli, reflecting interference due to the prior association with only stop trials. This slowing was expected to be greater in the presence of nicotine, again reflecting stronger associations between the "stop-go" face stimuli and the stop signal/stop response. Conversely, we expected greater speeding of goRTs for "go-go" stimuli relative to "go/stop-go" stimuli with nicotine.

Two task versions, each with a unique set of 50 face stimuli, enabled repeat-testing over the nicotine and the placebo session.

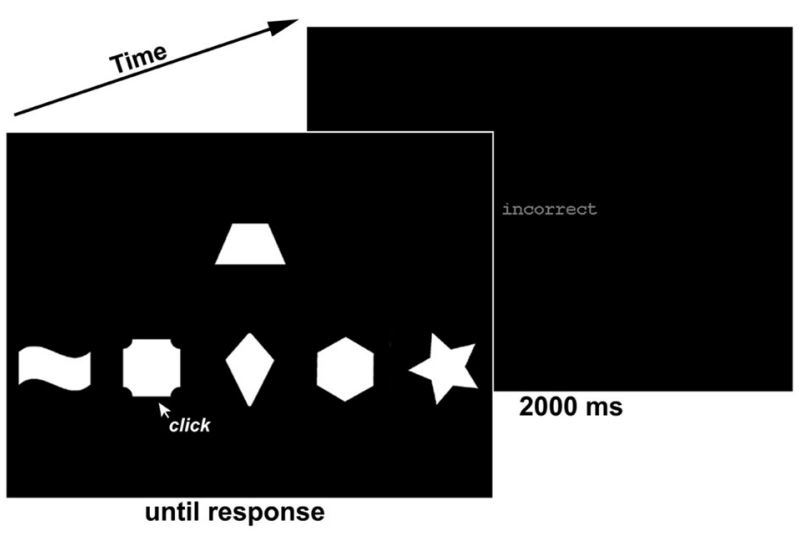

\begin{tabular}{|c|c|}
\hline \multicolumn{2}{|c|}{ Unique Task } \\
\hline $\begin{array}{c}\text { Test } \\
\text { stimulus }\end{array}$ & $\begin{array}{c}\text { Response } \\
\text { stimulus }\end{array}$ \\
\hline A & F \\
\hline B & G \\
\hline C & H \\
\hline D & I \\
\hline E & J \\
\hline
\end{tabular}

\begin{tabular}{|c|c|}
\hline \multicolumn{2}{|c|}{ Crossed Task } \\
\hline $\begin{array}{c}\text { Test } \\
\text { stimulus }\end{array}$ & $\begin{array}{c}\text { Response } \\
\text { stimulus }\end{array}$ \\
\hline A & C \\
\hline B & A \\
\hline C & E \\
\hline D & B \\
\hline E & D \\
\hline
\end{tabular}

Fig. 2 Task displays in the conditional associative learning task. The stimuli are shown to scale. The table lists possible stimulus-response mappings in the Unique task and Crossed task. Although the table utilizes the same letter placeholder, the two subtasks employed different shape stimuli from each other

Task versions were counterbalanced across drug conditions. Total task duration was $\sim 50 \mathrm{~min}$.

The conditional associative learning (CAL) task. The present task version was based on Gold et al. [46] (Fig. 2). Participants were presented with one out of five possible test stimuli in the top row, and five response stimuli in the bottom row of the screen display. They were asked to select, by mouse-click, the one response stimulus that "goes with" the test stimulus. The display was shown until response. All stimuli were shapes, white against a black background. Subjects initially guessed the correct response shape and, over time, learned the associations from feedback. Feedback was presented for $2 \mathrm{~s}$ after each response ("correct" written in green, or "incorrect" in red). An incorrect response was always followed by another presentation of the same display, until the correct response shape was selected. All incorrect choices within a trial were added and counted toward the total number of incorrect choices analyzed. The stimulus mappings never changed.

There were two subtasks, tested in counterbalanced order, employing different shape stimuli from each other (see table incorporated in Fig. 2):

(1) In the Unique task, there was a set of five test stimuli and a set of five response stimuli, consisting of all different shapes, i.e., there was no overlap between test and response stimuli. Each stimulus was part of one pair, creating five unique pairs.

(2) In the Crossed task, the test and response stimuli consisted of two identical sets of five shapes, and each test shape was associated with one of the four response shapes not identical to itself. Importantly, when shape A was the test stimulus, it may require the selection of response shape $C$, but when shape $C$ was the test stimulus, it required the selection of a response stimulus other than shape $A$ (shape $E$ in the example given in Fig. 2). Thus, forming a simple association between $A$ and $C$ would be insufficient for choosing the correct response shape; shape $C$ may be associated with either shape $A$ or shape $E$ depending on whether it was drawn from the test set or from the response set. Participants were fully aware of these rules. 
We predicted that, in the presence of nicotine, participants would make relatively more "context reversal errors" in the Crossed task. Context reversal errors are incorrect choices of response stimuli that were associated with the current test stimulus when the context was reversed, i.e., when they were themselves the test stimuli. An example from the table in Fig. 2 would be the choice of shape $A$ as the response stimulus when shape $C$ is the test stimulus, because shape $C$ "goes with" shape $A$ when shape $A$ is the test stimulus. More context reversal errors would demonstrate stronger incidental formation of stimulus-stimulus associations while failing to flexibly account for the context of the pairing.

Each subtask consisted of 40 trials. To verify learning with time on task, the total number of incorrect choices of any kind was quantified in each of four 10-trial blocks. Repeat-testing across the nicotine and placebo sessions was enabled by creating two CAL task versions, each with a unique set of 15 shape stimuli (10 shapes for each Unique task version and 5 shapes for each Crossed task version). Total task duration was $\sim 25 \mathrm{~min}$.

Data analysis

Stop-signal task

Training phase: SSDs were averaged across blocks 1-3 and across blocks 4-6 of the training phase for each stimulus type. SSDs averaged over "go/stop-go" and "go/stop-go/stop" stimuli (not systematically paired with stop or go trials in the training phase; 144 trials) were subtracted from SSDs averaged over "stop-go" stimuli (paired with stop trials; 36 trials). These difference values were analyzed by 3 -factor ANOVA for repeated measures with Drug (nicotine, placebo) and Block (1-3, 4-6) as within-subject factors and Drug sequence (nicotine tested before placebo, placebo tested before nicotine) as between-subjects factor, followed by paired $t$-tests where appropriate.

Transfer phase: goRTs of trials in which a correct male/female response was made were averaged over "stop-go" stimuli (previously paired with stop trials; 24 trials), "go-go" stimuli (previously paired with go trials; 48 trials), and "go/stop-go" stimuli (not previously paired with stop or go trials in a systematic manner; 32 trials). Average goRTs in "go/stop-go" trials were then subtracted from goRTs in "stop-go" trials and from goRTs in "go-go" trials. These difference values were compared between the nicotine and placebo session by 2 -factor ANOVA with Drug as within-subject factor and Drug sequence as between-subjects factor.

CAL task: To verify learning with time on task, 4-factor ANOVA was performed on the total number of incorrect choices, including within-subject factors Drug (nicotine, placebo), Subtask (unique, crossed), and Block $(1,2,3,4)$, and between-subjects factor Drug sequence. To test the main hypothesis, a 3-factor ANOVA was performed on the percentage of context reversal errors out of all incorrect choices made in the Crossed task. This ANOVA included within-subject factors Drug and Block, and between-subjects factor Drug sequence.

\section{RESULTS}

Stop signal task

Basic task performance: Thirty-one of the 32 participants inhibited between 40 and $60 \%$ of responses in stop trials, reflecting the dynamic performance-based adjustment of SSD to yield an average of $50 \%$ successful inhibitions. However, one participant inhibited only $7.6 \%$ of responses in stop trials and was excluded from analyses of this task. For another subject, the transfer phase in one of the test sessions could not be completed due to a technical error. This participant was excluded from analyses of the transfer phase, resulting in $N=30$ for these analyses, vs. $N=31$ for analysis of the training phase. The average accuracy of the male/ female discrimination was $97.7 \% \quad( \pm 1.9$ stdev $)$. All subjects responded with $\geq 91 \%$ accuracy.

Training phase. Fig. 3a shows the SSD difference values between "stop-go" stimuli, which were paired with stop trials, and stimuli that were not systematically paired with stop or go trials during training. Positive values indicate that stopping was facilitated by "stop-go" stimuli. In both test sessions, this facilitation was larger in the second half of the training (blocks 4-6) than in the first half (blocks 1-3), indicating associative learning between the "stop-go" stimuli and the stop signal/stop response with time on task. This was supported by a significant main effect of Block $[F(1,29)=5.24, P=0.030]$. There was no main effect of Drug
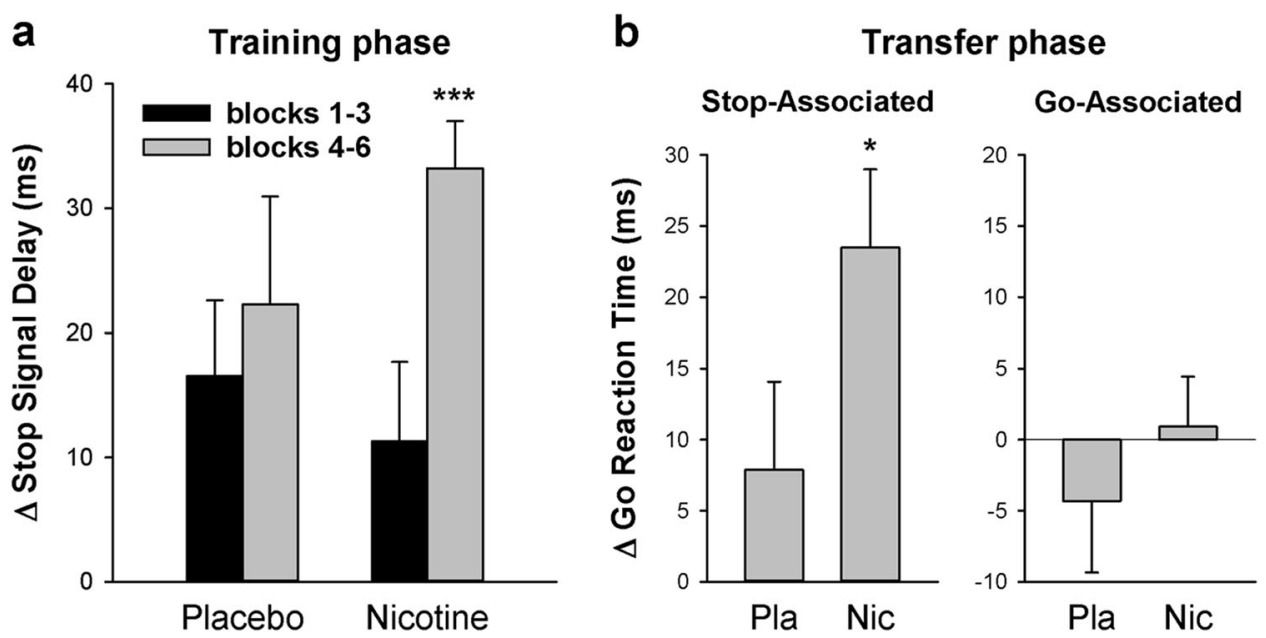

Fig. 3 a Average $( \pm$ SEM) Stop signal delay difference values between stimuli always paired with stop trials and stimuli not systematically paired with stop or go trials, in blocks $1-3$ and blocks $4-6$ of the training phase. ${ }^{* * *} P<0.001$, paired $t$-test comparing blocks $1-3$ with blocks 4-6 within session. b Average ( \pm SEM) go Reaction Time (goRT) difference values in the transfer phase between stimuli previously associated with stop signal trials (left) or go trials (right) and stimuli not previously associated with either stop or go trials in a systematic manner. Positive values reflect slower goRTs in response to stimuli previously associated with stop trials. Negative values reflect faster goRTs in response to stimuli previously associated with go trials. ${ }^{*} P<0.05$, paired $t$-test comparing the nicotine patch session (Nic) and placebo patch session (Pla) 

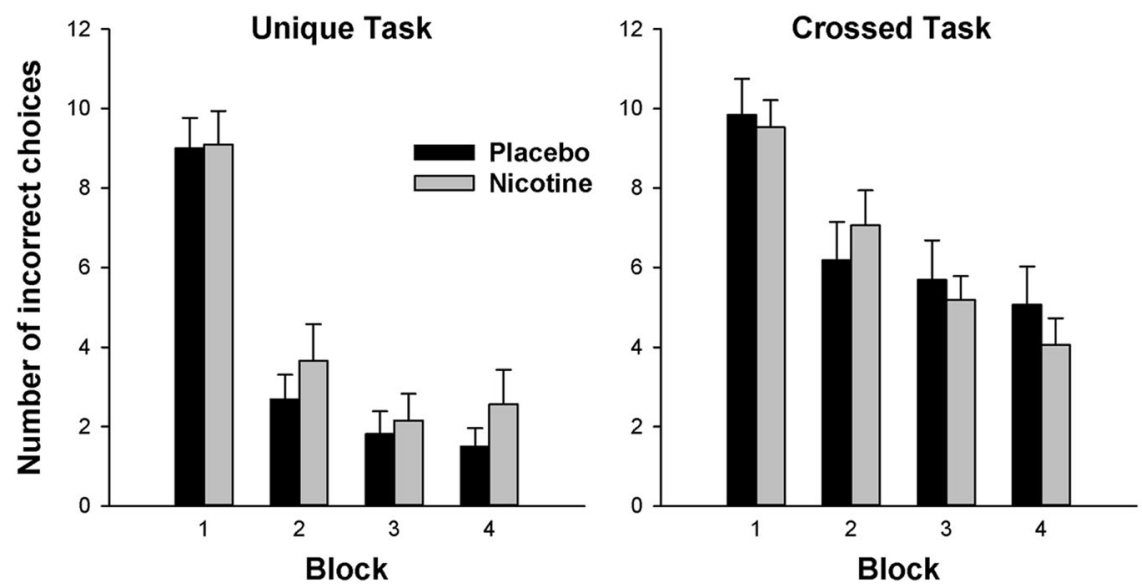

Fig. 4 Average $( \pm S E M)$ number of incorrect choices in the conditional associative learning task across blocks in the nicotine and placebo test session, for the Unique task (left) and the Crossed task (right)

$[F(1,29)=0.03, P>0.8]$. However, a significant Drug $\times$ Block interaction $[F(1,29)=4.44, P=0.044$; Cohen's $d=0.34]$ reflected an effect of Block in the presence of nicotine $[t(30)=3.93, P<0.001$; $d=0.75]$, but not in its absence $[P>0.5 ; d=0.14]$, consistent with facilitation of stimulus-stimulus or stimulus-response associative learning by nicotine. There were no main effects or interactions involving Drug sequence [all Ps $>0.25$ ].

Transfer phase. Fig. 3b, left graph, shows the goRT difference values between "stop-go" and "go/stop-go" stimuli. Positive values reflect slower goRTs to stimuli previously associated with stop trials. This slowing was significantly greater than zero in the nicotine session $[t(29)=4.29, P<0.001$; one-sample $t$-test $]$ but not in the placebo session $[t(29)=1.28, P=0.21]$. A significant main effect of drug $[F(1,28)=4.37, P=0.046$; Cohen's $d=0.49]$ in 2factor ANOVA confirmed that slowing was significantly greater in the presence of nicotine than in its absence. The main effect of drug sequence and the drug $\times$ drug sequence interaction were not significant $[P s>0.4]$.

Figure $3 b$, right graph, shows the goRT difference values between "go-go" and "go/stop-go" stimuli. Negative values would reflect faster goRTs to stimuli that had always been associated with go trials. However, the difference values did not differ from zero in either the nicotine session $[t(29)=0.26, P>0.7]$ or placebo session $[P=0.4]$, and there was no difference between sessions [main effect of drug: $F(1,28)=1.12, P=0.30$ ]. The main effect of drug sequence and the drug $\times$ drug sequence interaction were also not significant $[P S>0.3]$.

\section{CAL task}

Figure 4 displays the total number of incorrect choices with time on task in the nicotine and placebo session. For both the Unique task (left graph) and the Crossed task (right graph), incorrect choices declined across blocks, giving rise to a significant main effect of Block in 3-factor ANOVA $[F(3,90)=113.9, P<0.001]$. In the Unique task, this learning effect manifested itself as a sharp drop in incorrect choices after the first block. Performance improved more gradually across blocks in the Crossed task [block $\times$ subtask interaction: $F(3,90)=12.8, P<0.001]$. The presence of nicotine had no effect on incorrect choices; neither the main effect of drug, nor interactions involving drug were significant (all $P s \geq 0.4$ ). Furthermore, there was no main effect of drug sequence $[P>0.9]$ and no interactions involving drug sequence $[P s>0.11]$.

The total number of incorrect choices per session was higher in the Crossed task $(26.3 \pm 14.7$; mean \pm stdev) than in the Unique task $(14.2 \pm 12.4)$, as confirmed by a significant main effect of
Subtask $[F(1,30)=33.1, P<0.001]$. The total number of context reversal errors $(10.1 \pm 5.4)$, which are part of the incorrect choices in the Crossed task but cannot occur in the Unique task, numerically accounts for this difference.

Figure 5, top graph, displays the percentage of context reversal errors out of all incorrect choices in the Crossed task. The proportion of context reversal errors did not change with time on
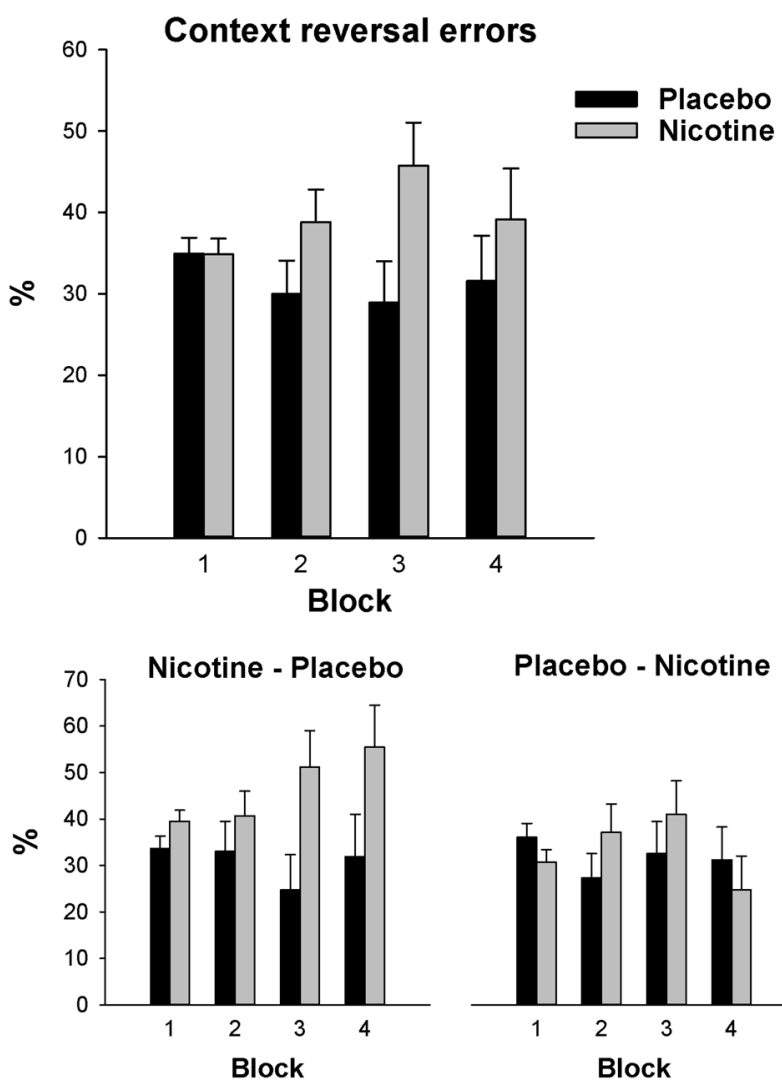

Placebo - Nicotine

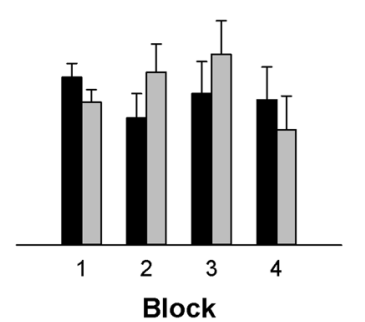

Fig. 5 Average $( \pm S E M)$ percentage of context reversal errors out of all incorrect choices made in the Crossed task, across blocks, in the nicotine session and the placebo session. The bottom graph on the left shows the data for participants receiving nicotine in the first test session and placebo in the second $(N=15)$, the graph on the right shows data for participants receiving placebo first and nicotine second $(N=17)$ 
task [main effect of block: $F(3,90)=0.16, P>0.9$ ]; however, as predicted, it was significantly larger in the nicotine session than in the placebo session [main effect of drug: $F(1,30)=6.29, P=0.018$; Cohen's $d=0.53$ ]. This difference was not seen in the first task block but emerged in later blocks. However, the drug $\times$ block interaction was not significant $[F(3,90)=1.70, P=0.174]$. There was no main effect of drug sequence $[F(1,30)=2.28, P=0.141]$, but the drug $\times$ drug sequence interaction was significant $[F(1,30)$ $=4.19, P=0.049$ ]. As illustrated by the bottom graphs in Fig. 5, the drug effect was significant only in individuals tested with nicotine first and placebo second $[F(1,14)=9.39, P=0.008]$, but not in individuals tested with the inverse sequence $[F(1,16)=0.12$, $P>0.7]$.

\section{DISCUSSION}

Traditionally, research on the cognitive effects of nicotine has focused on effects that facilitate functioning and may have therapeutic potential. Here, in contrast, we show that nicotine enhances learning mechanisms that can deepen the habitual and compulsive aspects of nicotine dependence. In a proof of concept study first of its kind in human subjects, we isolated the effects of nicotine on associative learning, the mechanism underlying the formation of drug-associated stimuli and conditioned responses. Several lines of evidence suggest that the behavioral control exerted by conditioned cues and response routines are of particular importance to tobacco dependence relative to other abused drugs [18, 19, 37], and the present results provide a potential explanation.

In the Stop signal paradigm, nicotine facilitated the formation of associations between previously neutral stimuli and the stop-signal/stop response. These associations manifested themselves twofold: (1) in a facilitated stop response to stop-associated stimuli, and (2) in a slowed go response when previously stopassociated stimuli were paired with go trials. While most face stimuli could coincide with stop trials, only a few (6 out of 50, randomly selected) did so in a systematic manner, and participants were not informed of this. Thus, these learning processes could not have unfolded in a deliberate, planned manner but were incidental. Most likely, they also occurred without awareness, but this is of no relevance for the purposes of the present study and was not verified.

In the CAL task, nicotine strengthened the association between two shape stimuli in a manner which, again, was indicative of incidental learning. When participants purposefully endeavored to learn which stimuli belonged together, nicotine did not facilitate the decline in errors across blocks. However, nicotine specifically increased the incorrect choice of stimuli that were associated with the test stimulus in a different context (i.e., when they were themselves the test stimuli). This effect only unfolded after the first task block, that is, not before any associations could have formed, although the drug $\times$ block interaction did not reach significance. An interaction with the sequence in which the drug conditions were tested suggested that the effect may have been weakened by greater awareness of this error source in the second test session. Overall, the effect reflects stronger stimulus-stimulus associations; however, it was maladaptive for task performance and occurred despite participants' knowledge of the task rules. Thus, facilitation of associative learning occurred at the expense of flexible context-adaptive behavior, mimicking the behavioral control by drug-associated stimuli which is thought to unfold in an automatized manner at the expense of frontoexecutive evaluative control [47-49].

While the above effects were of at most moderate size, there is strength in the fact that three out of four predictions consistent with enhanced associative learning were confirmed across two very different task paradigms. The only prediction not confirmed was that nicotine would enhance the acceleration of go responses to stimuli paired with go trials. No such acceleration was observed in either the nicotine or the placebo session, consistent with Lenartowicz et al. [42] and Verbruggen and Logan [43], and perhaps expectable given that the basal probability of go trials across all stimuli was $76 \%$. Thus, it appears that associative learning cannot take place under these conditions of limited predictiveness. Floor effects may also have prevented goRT acceleration in this speeded response task.

While the neuronal underpinnings of nicotine-induced facilitation of associative learning were not addressed by the present study, some speculation may be warranted on the basis of previous research. Early theories suggested that pairing with phasic drug-induced accumbal dopamine release is critical for the formation of drug-associated cues, by conferring incentive salience onto stimuli and behaviors associated with drug acquisition (reviewed by Ref. [37]). Theories proposing a central role of hippocampal functions, in contrast, are more specific to nicotine and are primarily based on preclinical studies of learning and memory outside of the addiction domain [40]. According to this view, nicotine facilitates hippocampus-based learning mechanisms more generally, and these effects were proposed to underlie the formation of maladaptive drug-cue and drug-context associations, thereby contributing to cuecontrolled drug-taking behavior [50]. While these theories are not mutually exclusive, our results support the view that nicotine's facilitation of a "cold", possibly hippocampusmediated cognitive process contributes to the behavioral control exerted by smoking-associated stimuli. However, data on potential neuronal mediators to date are based on rodent models, and little is known about the translatability between, for example, fear conditioning in rodents and multi-trial incidental association learning paradigms in humans.

Smoking-associated cues are multiform and do not necessarily represent discrete, clearly identifiable objects or stimuli. Any endogenous or exogenous state or state change systematically accompanying nicotine intake may become conditioned and acquire behavioral control. Conversely, the nicotine stimulus and smoking routine may acquire additional appetitive value through pairing with non-drug rewards such as relaxation, social interaction, or completion of a meal. The complexity of the conditioning processes that may contribute to dependent drug use is easily underestimated. Thus, the finding that nicotine can strengthen these processes is likely to be of great significance for understanding nicotine dependence, namely as a disorder of maladaptive associative learning.

The present finding that nicotine facilitates the formation of stimulus-stimulus and perhaps also stimulus-response associations at the expense of flexible context-adaptive behavior lays the groundwork for studies aimed at demonstrating the direct contribution of enhanced associative learning to dependent smoking. This endeavor should start with a replication of the present finding in dependent smokers (but bearing in mind the lack of a neutral baseline in nicotine-free smokers), a population that may inherently differ from non-smokers. Furthermore, to show that facilitation of associative learning contributes to nicotine dependence, future studies may test whether this effect generalizes to stimuli associated with the smoking routine and to self-administered nicotine.

\section{ACKNOWLEDGEMENTS}

We thank Drs. James Gold and lan Stolerman for helpful comments on the manuscript, and Hassan Omar for help with Matlab programming.

\section{FUNDING}

This work was funded by R03 DA037509 (B. Hahn). 


\section{ADDITIONAL INFORMATION}

Supplementary Information accompanies this paper at (https://doi.org/10.1038/ s41386-018-0183-9).

Competing interests: The authors declare no competing interests.

Publisher's note: Springer Nature remains neutral with regard to jurisdictional claims in published maps and institutional affiliations.

\section{REFERENCES}

1. Yoon PW, Bastian B, Anderson RN, Collins JL, Jaffe HW, Centers for Disease C. et al. Potentially preventable deaths from the five leading causes of death-United States, 2008-2010. MMWR Morb Mortal Wkly Rep. 2014;63:369-74.

2. Lopez AD, Mathers CD, Ezzati M, Jamison DT, Murray CJ. Global and regional burden of disease and risk factors, 2001: systematic analysis of population health data. Lancet. 2006;367:1747-57.

3. Giovino GA, Mirza SA, Samet JM, Gupta PC, Jarvis MJ, Bhala N, et al. Tobacco use in 3 billion individuals from 16 countries: an analysis of nationally representative cross-sectional household surveys. Lancet. 2012;380:668-79.

4. Messer K, Trinidad DR, Al-Delaimy WK, Pierce JP. Smoking cessation rates in the United States: a comparison of young adult and older smokers. Am J Public Health. 2008;98:317-22.

5. Stolerman IP, Jarvis MJ. The scientific case that nicotine is addictive. Psychopharmacol. 1995;117:2-10. Discussion14-20.

6. Anthony JC, Warner LA, Kessler RC. Comparative epidemiology of dependence on tobacco, alcohol, controlled substances, and inhalants: basic findings from the national comorbidity survey. Exp Clin Psychopharmacol. 1994;2:244-68.

7. Rose JE, Corrigall WA. Nicotine self-administration in animals and humans: similarities and differences. Psychopharmacol. 1997;130:28-40.

8. Manzardo AM, Stein L, Belluzzi JD. Rats prefer cocaine over nicotine in a two-lever self-administration choice test. Brain Res. 2002;924:10-9.

9. Freeman KB, Woolverton WL. Self-administration of cocaine and nicotine mixtures by rhesus monkeys. Psychopharmacol. 2009;207:99-106.

10. Mello NK, Newman JL. Discriminative and reinforcing stimulus effects of nicotine, cocaine, and cocaine + nicotine combinations in rhesus monkeys. Exp Clin Psychopharmacol. 2011;19:203-14.

11. Perkins KA, Grobe JE, Stiller RL, Fonte C, Goettler JE. Nasal spray nicotine replacement suppresses cigarette smoking desire and behavior. Clin Pharmacol Ther. 1992;52:627-34.

12. Benowitz NL, Zevin S, Jacob $P$ 3rd. Suppression of nicotine intake during ad libitum cigarette smoking by high-dose transdermal nicotine. J Pharmacol Exp Ther. 1998;287:958-62.

13. Donny EC, Jones M. Prolonged exposure to denicotinized cigarettes with or without transdermal nicotine. Drug Alcohol Depend. 2009;104:23-33.

14. Barrett SP, Darredeau C. The acute effects of nicotine on the subjective and behavioural responses to denicotinized tobacco in dependent smokers. Behav Pharmacol. 2012;23:221-7.

15. Butschky MF, Bailey D, Henningfield JE, Pickworth WB. Smoking without nicotine delivery decreases withdrawal in 12-hour abstinent smokers. Pharmacol Biochem Behav. 1995;50:91-6.

16. Di Chiara G. Behavioural pharmacology and neurobiology of nicotine reward and dependence. In: Clementi F, Fornasari D, Gotti C, (eds). Neuronal Nicotinic Receptors. Heidelberg: Springer; 2000. p. 603-50. Vol 144

17. Aubin HJ, Luquiens A, Berlin I. Pharmacotherapy for smoking cessation: pharmacological principles and clinical practice. Br J Clin Pharmacol. 2014;77:324-36.

18. Caggiula AR, Donny EC, White AR, Chaudhri N, Booth S, Gharib MA, et al. Cue dependency of nicotine self-administration and smoking. Pharmacol Biochem Behav. 2001;70:515-30.

19. Rose JE. Nicotine and nonnicotine factors in cigarette addiction. Psychopharmacol. 2006:184:274-85.

20. Droungas A, Ehrman RN, Childress AR, O'Brien CP. Effect of smoking cues and cigarette availability on craving and smoking behavior. Addict Behav. 1995;20:657-73.

21. Heishman SJ, Saha S, Singleton EG. Imagery-induced tobacco craving: duration and lack of assessment reactivity bias. Psychol Addict Behav. 2004;18:284-8.

22. Sayette MA, Martin CS, Wertz JM, Shiffman S, Perrott MA. A multi-dimensional analysis of cue-elicited craving in heavy smokers and tobacco chippers. Addiction. 2001;96:1419-32.

23. Abrams DB, Monti PM, Carey KB, Pinto RP, Jacobus SI. Reactivity to smoking cues and relapse: two studies of discriminant validity. Behav Res Ther. 1988;26:225-33.
24. Carter BL, Tiffany ST. Meta-analysis of cue-reactivity in addiction research. Addiction. 1999;94:327-40.

25. Tiffany ST, Cox LS, Elash CA. Effects of transdermal nicotine patches on abstinence-induced and cue-elicited craving in cigarette smokers. J Consult Clin Psychol. 2000;68:233-40.

26. Waters AJ, Feyerabend C. Determinants and effects of attentional bias in smokers. Psychol Addict Behav. 2000;14:111-20.

27. Waters AJ, Shiffman S, Bradley BP, Mogg K. Attentional shifts to smoking cues in smokers. Addiction. 2003;98:1409-17.

28. Bradley $B P$, Mogg $K$, Wright $T$, Field M. Attentional bias in drug dependence: vigilance for cigarette-related cues in smokers. Psychol Addict Behav. 2003;17:66-72.

29. Due DL, Huettel SA, Hall WG, Rubin DC. Activation in mesolimbic and visuospatial neural circuits elicited by smoking cues: evidence from functional magnetic resonance imaging. Am J Psychiatry. 2002;159:954-60.

30. Smolka MN, Buhler M, Klein S, Zimmermann U, Mann K, Heinz A, et al. Severity of nicotine dependence modulates cue-induced brain activity in regions involved in motor preparation and imagery. Psychopharmacol. 2006;184:577-88.

31. Brody AL, Mandelkern MA, London ED, Childress AR, Lee GS, Bota RG, et al. Brain metabolic changes during cigarette craving. Arch Gen Psychiatry. 2002;59:1162-72.

32. Shiffman S. A cluster-analytic classification of smoking relapse episodes. Addict Behav. 1986;11:295-307.

33. Shiffman S, Paty JA, Gnys M, Kassel JA, Hickcox M. First lapses to smoking: withinsubjects analysis of real-time reports. J Consult Clin Psychol. 1996;64:366-79.

34. Caggiula AR, Donny EC, White AR, Chaudhri N, Booth S, Gharib MA, et al. Environmental stimuli promote the acquisition of nicotine self-administration in rats. Psychopharmacol. 2002;163:230-7.

35. Wing VC, Shoaib M. Contextual stimuli modulate extinction and reinstatement in rodents self-administering intravenous nicotine. Psychopharmacol. 2008;200:357-65.

36. Palmatier MI, Evans-Martin FF, Hoffman A, Caggiula AR, Chaudhri N, Donny EC, et al. Dissociating the primary reinforcing and reinforcement-enhancing effects of nicotine using a rat self-administration paradigm with concurrently available drug and environmental reinforcers. Psychopharmacol. 2006;184:391-400.

37. Balfour DJ, Wright $A E$, Benwell ME, Birrell CE. The putative role of extra-synaptic mesolimbic dopamine in the neurobiology of nicotine dependence. Behav Brain Res. 2000;113:73-83.

38. Davis JA, Kenney JW, Gould TJ. Hippocampal alpha4beta2 nicotinic acetylcholine receptor involvement in the enhancing effect of acute nicotine on contextual fear conditioning. J Neurosci. 2007;27:10870-7.

39. Gould TJ, Higgins JS. Nicotine enhances contextual fear conditioning in C57BL/6J mice at 1 and 7 days post-training. Neurobiol Learn Mem. 2003:80:147-57.

40. Kenney JW, Gould TJ. Modulation of hippocampus-dependent learning and synaptic plasticity by nicotine. Mol Neurobiol. 2008:38:101-21.

41. Zhang TA, Tang J, Pidoplichko VI, Dani JA. Addictive nicotine alters local circuit inhibition during the induction of in vivo hippocampal synaptic potentiation. J Neurosci. 2010;30:6443-53.

42. Lenartowicz A, Verbruggen F, Logan GD, Poldrack RA. Inhibition-related activation in the right inferior frontal gyrus in the absence of inhibitory cues. J Cogn Neurosci. 2011;23:3388-99.

43. Verbruggen F, Logan GD. Automatic and controlled response inhibition: associative learning in the go/no-go and stop-signal paradigms. J Exp Psychol Gen. 2008:137:649-72.

44. Hannula DE, Ryan JD, Tranel D, Cohen NJ. Rapid onset relational memory effects are evident in eye movement behavior, but not in hippocampal amnesia. J Cogn Neurosci. 2007;19:1690-705.

45. Logan GD, Cowan WB, Davis KA. On the ability to inhibit simple and choice reaction time responses: a model and a method. J Exp Psychol Hum Percept Perform. 1984;10:276-91.

46. Gold JM, Bish JA, lannone VN, Hobart MP, Queern CA, Buchanan RW. Effects of contextual processing on visual conditional associative learning in schizophrenia. Biol Psychiatry. 2000;48:406-14.

47. Everitt BJ, Robbins TW. Drug addiction: updating actions to habits to compulsions ten years on. Annu Rev Psychol. 2016;67:23-50.

48. Hogarth L, Field M, Rose AK. Phasic transition from goal-directed to habitual control over drug-seeking produced by conflicting reinforcer expectancy. Addict Biol. 2013;18:88-97.

49. Tiffany ST. A cognitive model of drug urges and drug-use behavior: role of automatic and nonautomatic processes. Psychol Rev. 1990;97:147-68.

50. Davis JA, Gould TJ. Associative learning, the hippocampus, and nicotine addiction. Curr Drug Abus Rev. 2008;1:9-19. 